\title{
ON THE PENTADELTOID*
}

\author{
BY \\ R. P. S'TEPHENS
}

\section{INTRODUCTORY.}

In his Orthocentric Properties of the Plane n-Line, Professor MonLey $\dagger$ makes use of a curve of class $2 n-1$, which he calls the $\Lambda^{2 n-1}$. Of these curves, the deltoid $(n=2)$ is the well-known three-cusped hypocycloid; but the curve of class five $(n=3)$, which I shall call Pentadeltoid, and more frequently represent by the symbol $\Delta^{*}$, is not so well known, though its shape and a few of its properties were known to Clifford $\ddagger$. I shall here prove some of the properties of the pentadeltoid. My purpose in presenting this curve is twofold : 1 . the discussion of a curve of many interesting properties; 2 . the illustration of the use of vector analysis as a tool in the metric geometry of the plane.

The line equation of a curve is here uniformly expressed by meains of conjugate coïrdinates, $\S$ while the point equation is expressed by means of what is conmonly called the map equation, that is, a point of the curve is expressed as a function of a parameter $t$, which is limited to the unit circle and is, therefore, always equal in absolute value to unity. Throughout I shall consider $y$ and $b$ as conjugates of $x$ and $a$, respectively; that is, if $X$ and $Y$ are rectangular coördinates of a point, then

and similarly for $u$ and $b . \|$

$$
\begin{aligned}
& x=X+i Y, \\
& y=X-i Y,
\end{aligned}
$$

Some of the properties of the curves of class $2 n-1$ and of degree $2 n$, easily inferred from those of the $\Delta^{5}$, are also proved, but there has been no attempt at making a complete study of the general case.

\section{$\S 1$. The construction of the curvc.}

The line equation of the pentadeltoid, as given in Professor MorLey's article referred to above, is of the form

\footnotetext{
* Presented to the Society December 30, 1905. Received for publication September 15, 1905.

†Transactions of the American Mathematical Society, vol. 4 (1903), pp. 1-12.

†W. K. Clifford : Mathematical Papers, pp. 614-617, and plate opposite p. 642.

\$F. Frankin: Some cpplications of circular coördinctes, American Journal of Mathematics, vol. 12 (1890), p. 161. F. Mordey : On the Metric Geometry of the Plane n-Line, Transactions of the American Mathematical Society, vol. 1 (1900), p. 97.

$\|$ H. A. Converge : Annals of Mathematics, ser. 2, vol. 5 (1904), pp. 106-109, where, in a paper closely allied to this, is given a fuller explanation of these methods.
} 


$$
t^{5}+a t^{4}+x t^{3}+y t^{2}+b t+1=0,
$$

where $t$ is a turn and $a$ and $b$ are conjugates. For a fixed value of $t$, this is the equation of a line; but, as $t$ describes the unit circle, this line envelopes the $\Delta^{5}$.

The equation may be written in the form

$$
t x+y=t\left(-t^{2}-\frac{b}{t^{2}}\right)+\left(-\frac{1}{t^{2}}-a t^{2}\right)
$$

whence it is evident that it is a line with clinant $-1 / t$ through the point

$$
x_{1}=-t^{2}-\frac{b}{t^{2}} \text {. }
$$

For varying $t, x_{1}$ describes an ellipse with center at the origin; hence the $\Delta^{5}$ is the envelope of a line with clinant $-1 / t$ which passes through the point

$$
x_{1}=-t^{2}-\frac{b}{t^{2}}
$$

as $t$ varies. Or, putting it more concretely, we may say that if a straight edge be attached to the generating point of an ellipse and given the proper rotation about this point, its envelope will be a $\Delta$.

The ellipse can best be generated by means of the instrument commonly called the ellipsograph. The desired rotation of the straight edge can be secured by cog-wheels, properly geared. In the figure $1 A$, suppose the segment $A B$ of length 2 be allowed to move with its extremities always in the perpendicular lines $A O$ and $O B$, then the point $M$, the mid-point of $A B$, will trace out a unit circle. At any instant in its motion in a positive direction about $O$, let $M$ be at $\tau^{2}$; then, if $M P$ in absolute value is $\mu$, we have for the point $P$, since it revolves about $M$ in a negative direction at the same rate as $M$ about $O$,

$$
n_{1}=\tau^{2}+\frac{\mu}{\tau^{2}} .
$$

Centered at $A$ on bcth $A B$ and $A O$ is a cog-wheel $C_{3}$, which is so attached that its only motion is horizontal translation along $A O$. Centered on $A B$ is another cog-wheel $C_{4}$, which moves with $A B$, but also may rotate about $P$ as a center. These two cog-wheels, $C_{3}$ and $C_{4}$, whose diameters are in the ratio of 3 to 4 , are connected by the small cog-wheel $C$. The line $l$ passing through $P$ rotatess with $C_{4}$.

As $O M$ moves positively through an angle $\theta\left(\right.$ a turn $\left.\tau^{2}\right)$, the cog-wheel $C_{4}$, owing to the motion of $A B$, rotates with reference to the fixed plane through a negative angle $-\theta$; but, on account of its connection with $C_{3}$, it is turned in a positive direction $3 \theta$, which leaves it with a resultant rotation $-\frac{1}{4} \theta$, or a turn 
$1 / \sqrt{\tau}$. Hence, if the clinant of $l$ was initially $-\kappa$, it is now $-\kappa / \tau$. Therefore, since $l$ goes through the point

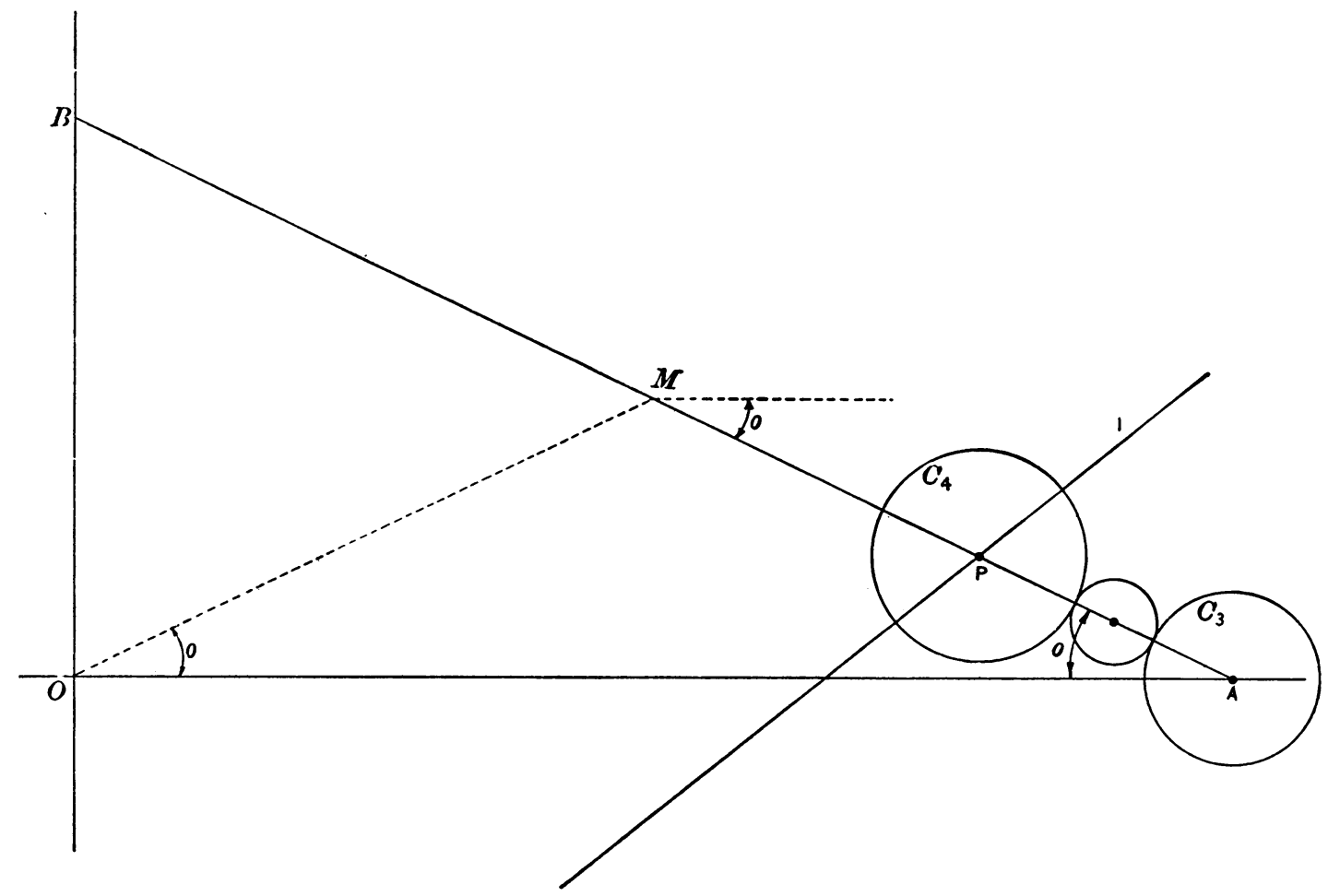

Fig. $1 A$.

$$
x_{1}=\tau^{2}+\frac{\mu}{\tau^{2}}
$$

its equation is

or putting

$$
x+\frac{\kappa}{\tau} y=\left(\tau^{2}+\frac{\mu}{\tau^{2}}\right)+\frac{\kappa}{\tau}\left(\frac{1}{\tau^{2}}+\mu \tau^{2}\right)
$$

$$
i t \equiv \tau \quad \text { and } \quad i \alpha \equiv \kappa,
$$

we have, as the equation of the line $l$,

$$
t^{5}+\alpha \mu t^{4}+x t^{3}+\alpha y t^{2}+\mu t+\alpha=0,
$$

where $t$ and $\alpha$ are turns and $\mu$ is real and positive. The two constants here, $\alpha$ and $\mu$, are of the sarne effect as the two conjugates, $a$ and $b$, used above, so this new equation is just as general as the first form.

If, in the mechanism described above, a cog-wheel $C_{5}$ be used for $C_{3}$, that is, if cog-wheels whose diameters have the ratio 5 to 4 instead of 3 to 4 be used, the equation of the curve will have the form 


$$
\mu t^{5}+\alpha t^{4}+x t^{3}+\alpha y t^{2}+t+\alpha \mu=0 .
$$

The advantage of this double description is easily seen. If $\mu=0$, then the first form becomes

$$
t^{5}+x t^{3}+\alpha y t^{2}+\alpha=0,
$$

which, as $t$ varies, envelopes the curve called by Clifford the five-rayed star, but which I shall call a regular $\Delta^{5}$. However, if $\mu=0$ in the second form, we have

$$
x t^{3}+x t^{2}+x y t+1=0,
$$

which is the line equation of the deltoid. So with the two combinations of cogwheels, which can be adjusted in the same mechanism, we can get all shapes of the $\Delta^{5}$ as it varies from the five-rayed star to the deltoid.

The diagram of Fig. $1 B$ shows the instrument with which the curves in the other figures were described.

\section{\$2. The equation of the curre.}

The $\Delta^{i}$ as here treated has the equation

$$
t^{3}+\alpha \mu t^{4}+x t^{3}+\alpha y t^{2}+\mu t+\alpha=0 .
$$

If we divide this equation by $t^{2}$ and then differentiate as to $t$, we shall obtain the map equation of the curve. Thus

$$
x=-\left(3 t^{2}+2 \alpha \mu t-\mu-\begin{array}{c}
2 x \\
t^{2}
\end{array}-\begin{array}{c}
t^{3}
\end{array}\right),
$$

where $t$ is the variable turn. Evidently for any given value of $t,(2)$ is that point of the curve at which the line (1) is tangent.

From equation (1) it is seen that from any point $x$ there are five tangents to the curve; that is, it is of the fifth class. By substituting $x$ and its conjugate from equation $(2)$ in the equation of a line, we obtain a sextic in $t$; hence the curve is of the sixth order.

Again, since the sum of five turns can never be greater in absolute value than 5 , it is seen from (1), that if $\mu>5$, there is no point of the plane from which five tangents, all of them real, can be drawn to the curve.

Whenever reference is made to the general case, ${ }^{*}$ the equation of the curve of class $4 n-1$ will be assumed of the form :

$$
\begin{aligned}
x t^{4 n-1}+a_{1} t^{4 n-2}+x u_{2} t^{t n-3}+\cdots & +\alpha \mu t^{2 n+1}+x t^{2 n} \\
& +\alpha y t^{2 n-1}+\mu t^{2 n-2}+\cdots+b_{2} t^{2}+\alpha b_{1} t+1=0 ;
\end{aligned}
$$

*F. MoRLky : Transactions, loc. cit. 
and of class $4 n+1$ :

$$
\begin{aligned}
& t^{4 n+1}+\alpha a_{1} t^{t n}+a_{2} t^{t n-1}+\cdots+\alpha \mu t^{2 n+2}+x t^{2 n+1} \\
& +\alpha y t^{2 n-}+\mu t^{2 n-1}+\cdots+\alpha b_{2} t^{2}+b_{1} t+\alpha=0,
\end{aligned}
$$

where $\alpha$ and $t$ are turns, $\mu$ is real, and $a_{i}$ and $b_{i}$ are conjugates. These forms

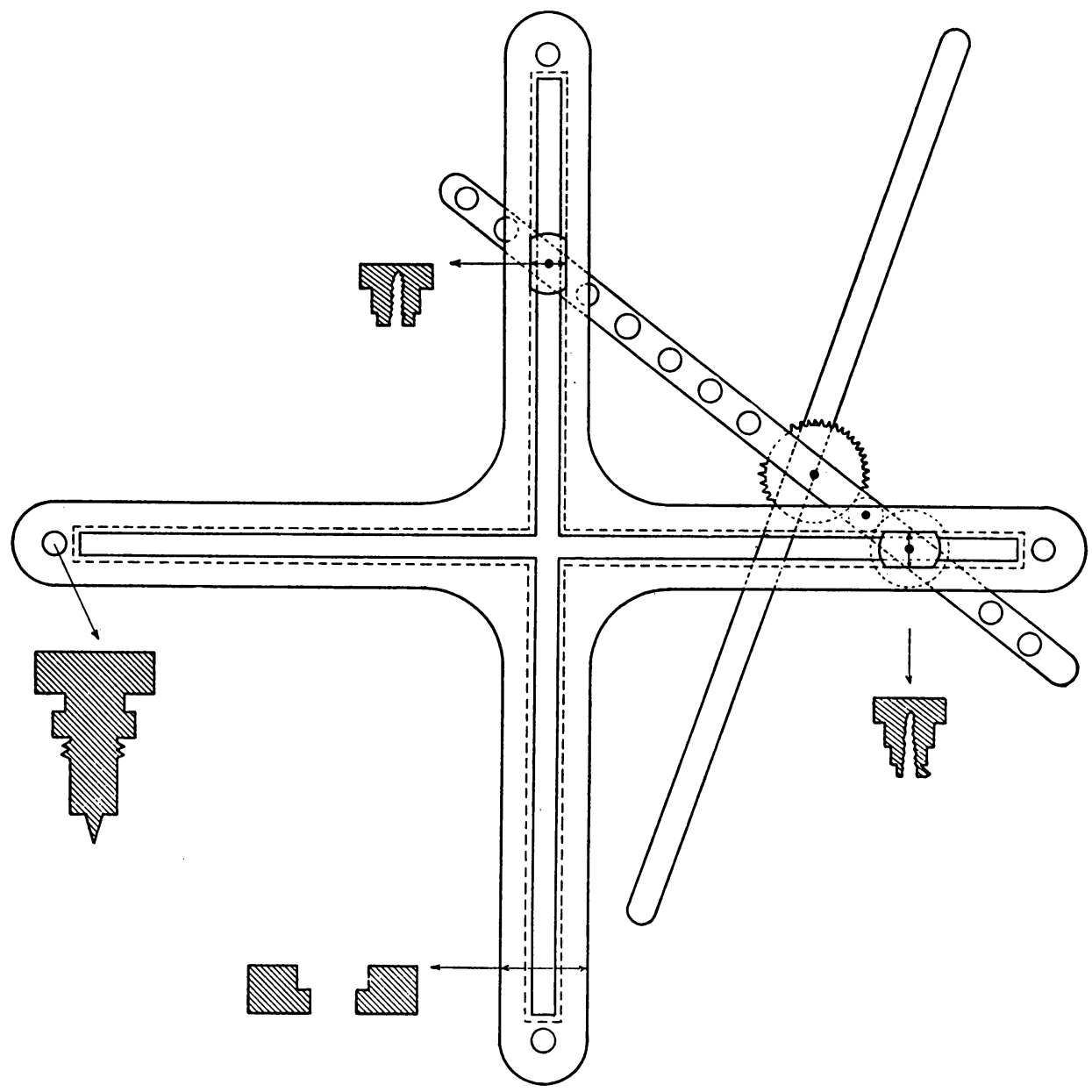

Fia. $1 B$.

are derived in a manner analogous to the case of the $\Delta^{5}$. The curve $\Delta^{7}$, for instance,

$$
t^{7}+a_{1} t^{6}+a_{2} t^{5}+x t^{4}+y t^{3}+b_{2} t^{2}+b_{1} t+1=0,
$$

can be written in the form

$$
t x+y=-t\left(a_{1} t^{2}+\frac{b_{2}}{t^{2}}+\frac{1}{t^{4}}\right)-\left(\begin{array}{l}
b_{1} \\
t^{2}
\end{array}+a_{2} t^{2}+t^{4}\right),
$$


obviously a line through the point

$$
x=-\left(a_{1} t^{2}+\begin{array}{c}
b_{2} \\
t^{2}
\end{array}+\frac{1}{t^{4}}\right) .
$$

By a proper choice of a unit circle, one of the two complex constants, $a_{1}$ s.nd $b_{2}$, can be made real, then we have

$$
x=-\left(a t^{2}+\frac{\mu}{t^{2}}+\frac{1}{t^{4}}\right)
$$

where $\mu$ is real. The $\Delta^{7}$ is, therefore, the envelope of a line through this point as $t$ varies, provided the line be given the proper rotation about this point. Owing to our change in the unit circle, we must take account of the initial inclination of the line and so must introduce a constant turn, $\alpha$. Therefore the equation of the line is

$$
\left.t x+\alpha y=-t\left(a t^{2}+\begin{array}{c}
\mu \\
t^{2}
\end{array}+\begin{array}{c}
1 \\
t^{4}
\end{array}\right)-\alpha\left(\begin{array}{c}
b \\
t^{2}
\end{array}\right) \mu t^{2}+t^{4}\right) .
$$

This may be put into the form

$$
\alpha t^{7}+a t^{6}+\alpha \mu t^{5}+x t^{4}+\alpha y t^{3}+\mu t^{2}+b t+\alpha=0
$$

a line which for varying $t$ envelopes the $\Delta^{?}$.

So in general we can replace one of the complex constants of the $\Delta^{2 n-1}$. when in the form given by Professor MorLey, by a real number and a turn,and thus obtain the general equations as given above.

\section{\$3. Perpendicular tangents.}

If equation (1) is a tangent to the curve, then obviously the tangent perpendicular to it is obtained by putting $-t$ for $t$ in (1). Thus,

$$
-t^{5}+\alpha \mu t^{4}-x t^{3}+\alpha y t^{2}-\mu t+\alpha=0 \text {. }
$$

The point of intersection of these two lines is

$$
x=-\left(t^{2}+\begin{array}{l}
\mu \\
t^{2}
\end{array}\right)
$$

which for varying $t$ is an ellipse, in fact the same ellipse which appeared above in the construction of the curve. Hence, perpendicular tangents of ' $\left(\Delta^{5}\right.$ intersect along an ellipse.

This ellipse, the orthoptic curve, has its center at the origin and its foci on the axis of reals. For $\mu=0$, it becomes the unit base circle and for $\mu=1$, it becomes the segment of a line. For convenience, I shall refer to the center of the orthoptic curve of the $\Delta^{5}$ as the center of that $\Delta^{5}$. 
That this ellipse is tangent to the $\Delta^{5}$ can be shown in this way. The clinant of the tangent to the ellipse at any point is

$$
\frac{d x}{d y}=\frac{\frac{d x}{d t}}{d y}=\frac{t^{t}-\mu}{\mu t^{4}-1}
$$

Evidently, one of the two perpendicuiar tangents to the $\Delta^{5}$ which intersect on the ellipse will be tangent to the ellipse at that point of intersection, provided its clinant equals the clinant above, that is, if

$$
\begin{gathered}
t^{4}-\mu \\
\mu t^{4}-1
\end{gathered}=-\stackrel{\alpha}{t}, \quad \text { or } \quad t^{5}+\alpha \mu t^{4}-\mu t-\alpha=0 .
$$

There are therefore five common tangents of the $\Delta^{5}$ and its orthoptic ellipse.

Now the points at which a common tangent touches the two curves have the same parameter $t$, and hence coincide if

This reduces to

$$
t^{2}+\frac{\mu}{t^{2}}=3 t^{2}+2 \alpha \mu t-\frac{\mu}{t^{2}}-\stackrel{2 \alpha}{t^{3}} .
$$

$$
t^{5}+\alpha \mu t^{4}-\mu t-\alpha=0,
$$

which is condition (4) above; so a common tangent touches the two curves in coincident points. Therefore, the $\Delta^{5}$ touches its orthoptic ellipse in five points. (See Fig. 2.)

In an exactly similar manner, the corresponding theorem may be proved for the $\Delta^{2 n-1}$, that is, the $\Delta^{2 n-1}$ is tangent to its orthoptic curve in $2 n-1$ points.

From (4), it is evident that at least two of the common tangents become imaginary for $\mu>5$. This' is a sufficient but not a necessary condition for imaginary common tangents.

The general contact theorem above could have been stated otherwise, thus: the $\Delta^{2 n-1}$ has $2 n-1$ tangents which are also normals not only to itself but also to its orthoptic curve. The equation giving the parameters of these normals in the case of the $\Delta^{5}$ is the result of substituting $-t$ for $t$ in equation (4); it is therefore

$$
t^{5}-\alpha \mu t^{4}-\mu t+\alpha=0 .
$$

If we subtract this from equation $(1)$, the line equation of the $\Delta^{5}$, we obtain

$$
2 \alpha \mu t^{3}+x t^{2}+\alpha y t+2 \mu=0,
$$

the line equation of a deltoid whose map equation is

$$
x=-2 \mu\left(2 \alpha t-\begin{array}{c}
1 \\
t^{2}
\end{array}\right) .
$$


From this it is seen that the five tangents of a $\Delta^{5}$ which are also normals to itself and its orthoptic ellipse touch a concentric deltoid.

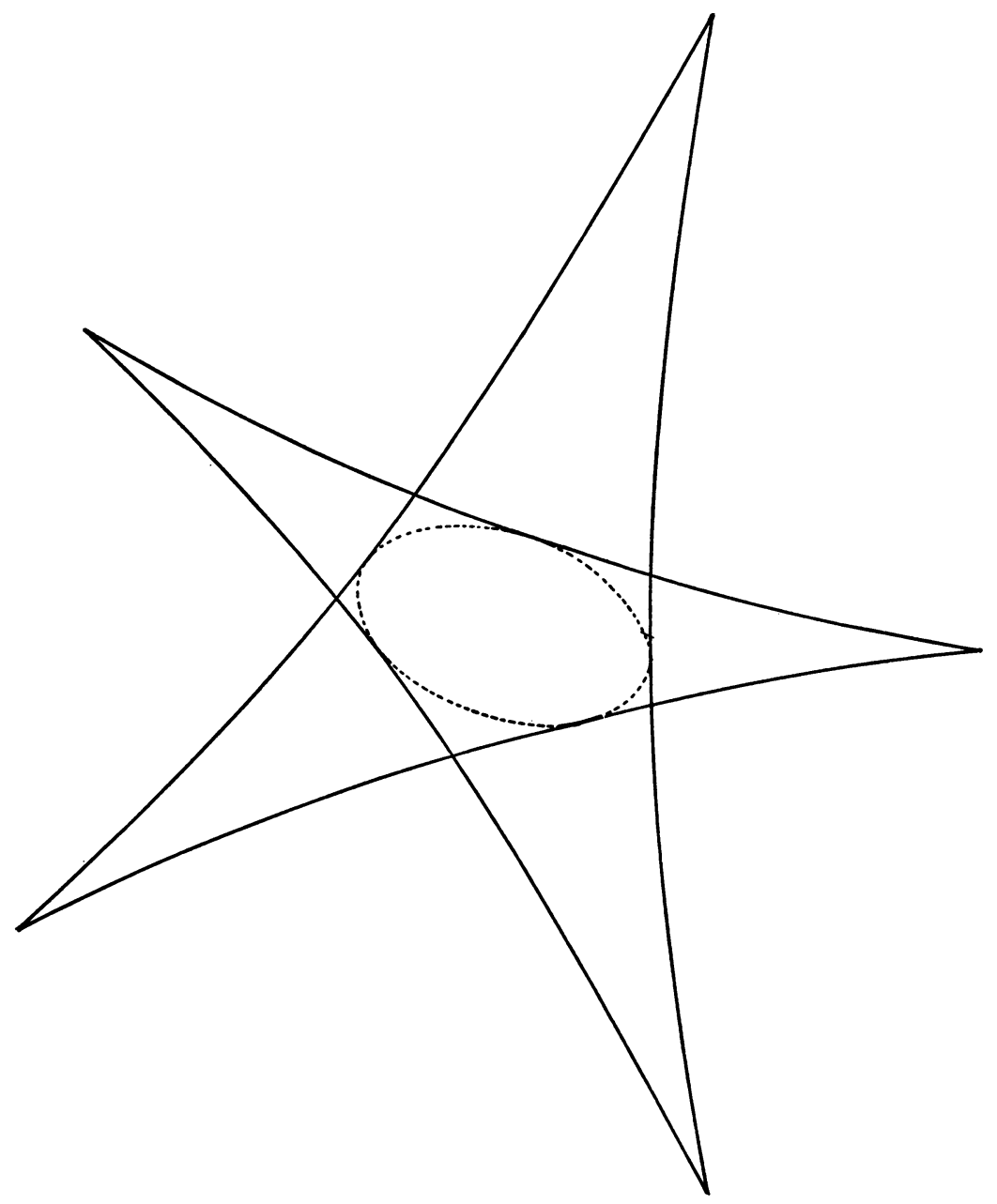

FiG. 2. Pentadeltoid when $\mu=.2$.

In a similar manner, the $2 n-1$ tangents of a $\Delta^{2 n-1}$ which are normals common to itself and its orthoptic curve touch a concentric $\Delta^{2 n-3}$.

If equation ( 5 ) be added to ( 1 ), then

or

$$
2 t^{5}+x t^{3}+\alpha y t^{2}+2 \alpha=\hat{0},
$$

$$
x=-2\left(3 t^{2}-\frac{2 \alpha}{t^{3}}\right),
$$

the equation of a regular $\Delta^{5}$, which is independent of $\mu$. Helce, the five tan- 
gents of a $\Delta^{5}$ which are normals conmon to itself and its orthoptic ellipse touch a concentric regular $\Delta^{5}$; and such normals, for a system of $\Delta^{53} s$ having the same $\alpha$, envelope the same regular $\Delta^{5}$ for a varying $\mu$.

There is an interesting generalization suggested by the numbers required to determine the orthoptic curve of the deltoid and the pentadeltoid. In each case the number of points of contact of the orthoptic curve and the $\Delta^{3}$ or $\Delta^{5}$ is just sufficient to determine the orthoptic curve. That this law holds in general is easily proved. Consider a curve of class $4 n-1$ given by $\alpha t^{4 n-1}+a_{1} t^{4 n-2}+\alpha a_{2} t^{4 n-3}+\cdots+\alpha \mu t^{2 n+1}+x t^{2 n}+\alpha y t^{2 n-1}+\cdots+\alpha b_{1} t+1=0$. Its orthoptic curve is obtained just as was that of the $\Delta^{5}$. It is

$$
x=-\left(a_{1} \tau^{n-1}+a_{3} \tau^{n-2}+\cdots+a_{2 n-3} \tau+\frac{\mu}{\tau}+\cdots+\frac{1}{\tau^{n}}\right),
$$

where $\tau \equiv t^{2}$. This is a curve of order $2 n$. In counting its constants we must remember that the base circle requires four constants and that each complex number carries with it its conjugate. Hence, the numbers for the orthoptic curve are :

Base circle

4 constants,

Constants $a_{1}, a_{3}, a_{5}, \ldots, a_{2 n-3}, 2\left[\frac{2 n-3+1}{2}\right]=2 n-2 \quad$ “ ,

Constants $b_{2}, b_{1}, \cdots, b_{2 n-4}, 2\left[\frac{2 n-4}{2}\right] \quad=2 n-4 \quad$ “ ,

Constant $\mu$, Total

$$
\frac{1}{4 n-1} \text { constants. }
$$

But we have seen that $4 n-1$ is the number of points of contact of the $\Delta^{4 n-1}$ and its orthoptic curve. In a similar manner, the curve,

$$
\begin{aligned}
t^{t n+1}+\alpha a_{1} t^{4 n}+a_{2} t^{4 n-1}+\cdots+\alpha \mu t^{2 n+2}+ & x t^{2 n+1} \\
& +\alpha y t^{2 n}+\mu t^{2 n-1}+\cdots+b_{1} t+\alpha=0,
\end{aligned}
$$

has for its orthoptic curve

$$
x=-\left(\tau^{n}+a_{z} \tau^{n-1}+\cdots+a_{2 n-2} \tau+\frac{\mu}{\tau}+\cdots+\frac{b_{1}}{\tau^{n}}\right),
$$

a curve of order $2 n$. Counting the constants here as in the other case, we find $4 n+1$, which again agrees with the number of points of contact. Therefore, in general, the points of tangency of a $\Delta^{2 n-1}$ with its orthoptic curve are just sufficient to determine that curve.

The degree of the orthoptic curve of the $\Delta^{t \mu-i}$ is the same as that of the orthoptic curve of the $\Delta^{4 n+1}$ - each is $2 n$. But in all cases the numbers 
necessary to determine the $\Delta^{2 n-1}$ are just one more than those required for its orthoptic curve. In the equations given this extra number is $\alpha$. From this comes an important theorem : Connected with the orthoptic curve of any $\Delta^{2 n-1}$ is a singly infinite system of $\Delta^{2 n-1}$ 's, all having the same orthoptic curve.

For the $\Delta^{5}$, this last theorem is seen directly. The inscribed ellipse (3) is obviously independent of $\alpha$, and hence there is a single infinity of $\Delta^{5} s$, each of which touches the same ellipse in five points.

\section{§4. Nodes and their locus.}

In a previous section it was pointed out that there are, in general, five tangents to a $\Delta^{3}$ from any point of the plane. From a node, however, two pairs of tangents coincide and so there are only three tangents. Hence, if

$$
t^{5}+\alpha \mu t^{4}+x t^{3}+\alpha y t^{2}+\mu t+\alpha=0
$$

be considered a polynomial in $t$, with roots $t_{i}$ (where $i=1,2, \ldots, 5$ ), then, at a node,

$$
t_{1}=t_{2}, \quad t_{3}=t_{4}
$$

Equating the symmetric functions of these roots to the coefficients of $(1)$, we have

where

$$
\begin{gathered}
2 \sigma_{1}+t=-\alpha \mu, \quad 2 \sigma_{1} t+\sigma_{1}^{2}+2 \sigma_{2}=x, \\
2 \frac{\sigma_{1}}{\sigma_{2}}+\frac{1}{t}=-\frac{\mu}{\alpha}, \quad \sigma_{2}^{2} t=-\alpha,
\end{gathered}
$$

$$
t \equiv t_{5}, \quad \sigma_{1} \equiv t_{1}+t_{3}, \quad \sigma_{2} \equiv t_{1} t_{3} .
$$

If now we put $t_{1} \equiv \sqrt{t}$ and $\kappa \equiv \sqrt{-\alpha}$ and eliminate $\sigma_{1}$ and $\sigma_{2}$ from the third equation by means of the first and fourth equations, we obtain

$$
\kappa t_{1}^{j}-\kappa^{3} \mu t_{1}^{3}+\mu t_{1}^{2}-\kappa^{2}=0 .
$$

Or by eliminating $\sigma_{1}$ and $\sigma_{2}$ from the second equation, with the help of the first and fourth, there results

$$
4 x=-3 t_{1}^{4}+2 \kappa^{2} \mu t_{1}^{2}+\kappa^{4} \mu^{2}+8 \frac{\kappa}{t_{1}} .
$$

Of the three tangents from a node, only one is not tangent at the node, this we shall call the odd tangent. Then equation (6) gives the odd tangents. Evidently there are, in general, five such tangents. If $\mu>10$, at least two of these tangents disappear.

Fquation (7) represents a curve on which the five nodes lie. By means of relation (6) it can be brought to a simpler form, viz.

$$
4 x=-\mu \frac{\kappa^{3}}{t_{1}^{3}}+\left(\mu^{2}+5\right) \frac{\kappa}{t_{1}}+3 \mu \frac{t_{1}}{\kappa} .
$$


Since it is always allowable to substitute one turn for another in the map equation of a curve, we can here put $\tau \equiv \kappa / t_{1}$ and thus obtain

$$
4 x=-\mu \tau^{3}+\left(\mu^{2}+5\right) \tau+\frac{3 \mu}{\tau} .
$$

This is the equation of a parastroid,* or parallel to an astroid, which is not changed by varying $x\left(=-\kappa^{2}\right)$; therefore, the locus of nodes of the system of $\Delta^{5}$ 's having an inscribed ellipse in common is a concentric parastroid.

The line equation of this curve is

$$
\tau^{+}-\frac{2}{\mu} x \tau^{3}+\left(\mu+\frac{5}{\mu}\right) \tau^{2}-\frac{2}{\mu} y \tau+1=0 .
$$

Since the coefficient of $\tau^{2}$ is a constant times the distance between a tangent of the parastroid and a corresponding parallel tangent of its astroid, the shape of (9) depends wholly on the value of $\mu+5 / \mu$. The curve is an astroid when

$$
\mu+\frac{5}{\mu}=0, \quad \text { or } \quad \mu=\sqrt{-5} \text {, }
$$

but by hypothesis $\mu$ is real, therefore (9) can never be an astroid. The curve is tangent to itself, if

$$
\mu+\frac{5}{\mu}= \pm 2
$$

but this equation again has no real roots. The real limitations of the curve (9), however, can best be seen from the equation giving the parameters of the cusps, which is obtained on equating to zero the derivative of $x$ : as to $\tau$ in equation (8). It is

$$
3 \mu \tau^{4}-\left(\mu^{2}+5\right) \tau^{2}+3 \mu=0 .
$$

Two cusps coincide when this has equal roots, that is, when

or

$$
\left(\mu^{2}-1\right)\left(\mu^{2}-25\right)=0,
$$

$$
\mu=1, \quad \mu=5 \text {. }
$$

So there are 5 cusps for $1<\mu<5$. For the limiting values, 1 and 5 , the cusps coincide by twos and we get the form $\dagger$ of the parastroid wbich closely resembles an ellipse whose axes are to each other as one to two. For $\mu>5$ and for $0<\mu<1$, the curve (9) has no cusps and varies in shape from the form just mentioned (where $\mu=1$ or 5 ) to the circle (where $\mu=0$ or $\infty$ ).

* G. Loria : Spezielle ebine Kurven, p. 651.

† Wolstenholme's Mathematical Problems, p. 303. 
The odd tangents from the nodes, we have seen, must satisfy relation (6) above. The clinant of any one of these lines is

$$
-\frac{\alpha}{t}=\frac{\kappa^{2}}{t_{1}^{2}}=\tau^{2}
$$

these turns having the same value as they had above. A clinant of a normal to the node locus (8) at a node is

$$
-d y=\frac{\tau^{2}\left[3 \mu \tau^{4}-\left(\mu^{2}+5\right) \tau^{2}+3 \mu\right]}{\left[3 \mu-\left(\mu^{2}+5\right) \tau^{2}+3 \mu \tau^{4}\right]}=\tau^{2} .
$$

Since the odd tangent and the normal to the node locus at a node have the same clinant and pass through the same point, they must coincide. So we may say that the five odd tangents of any $\Delta^{5}$ are normal to the locus of nodes of the system of $\Delta^{5}$ 's which have an inscribed ellipse in common.

\section{§5. Cusps, cusp loci, and cuspidal tangents.}

The map equation of the $\Delta^{5}$ is

$$
x=-\left(3 t^{2}+2 a \mu t-\frac{\mu}{t^{2}}-\frac{2 \alpha}{t^{3}}\right) .
$$

A cusp of this curve will be such a point, $x$, that for a change in $t$ there will be no change in $x$; or, borrowing a phrase from kinematics. a cusp is that point of the curve at which the generating point has zero velocity. The condition, therefore, is $d x / d t=0$. If the values of $t$ thus obtained are turns, then there are cusps. From (2) we get as the cusp equation

$$
3 t^{5}+\alpha \mu t^{4}+\mu t+3 \alpha=0,
$$

the roots of which are, in general, turns; hence we say the $\Delta^{5}$ has five cusps. If, however, $\mu>15$, at least two of these cusps must disappear.

If equation (10) be subtracted from three times equation (1), we get

or

$$
2 \alpha \mu t^{3}+3 x t^{2}+3 \alpha y t+2 \mu=0,
$$

$$
3 x=-2 \mu\left(2 \alpha t-\begin{array}{c}
1 \\
t^{2}
\end{array}\right)
$$

the equation of a concentric deltoid; that is, ${ }^{*}$ ihe five cusp-tangents of a $\Delta^{5}$ touch a concentric $\Delta^{3}$. And in general, by a similar argument, we see that the $2 n-1$ cusp-tangents of a $\Delta^{2 n-1}$ touch a concentric $\Delta^{2 n-3}$.

*'The proofs of these three theorems are due to Professor MorLey, v. Transactions of the American Mathematical Society, vol. 4 (1903), p. 8. The first two theorems are given by ClifFord, loc. cit. 
If $(10)$ be subtracted from $(1)$, there results

$$
2 t^{5}-x t^{3}-\alpha y t^{2}+2 \alpha=0,
$$

or

$$
x=2\left(3 t^{2}-\begin{array}{c}
\alpha \\
t^{3}
\end{array}\right),
$$

the equation of a concentric regular $\Delta^{5}$. The five cusp-tangents of a $\Delta^{5}$ touch a concentric regular $\Delta^{5}$. In a similar manner, the $2 n-1$ cusp-tangents of a $\Delta^{2 n-1}$, determined by $2 n$ constants, touch a concentric $\Delta^{2 n-1}$ determined by $2 n-2$ constants.

The cusp locus for the system of $\Delta^{5}$ 's, having the same inscribed ellipse, is obtained by eliminating $\alpha$ between equations $(10)$ and (2). It is

or

$$
x=-6 t^{2}+\frac{\left(3 t^{4}+\mu\right)\left(3 \mu t^{4}+1\right)}{\left(\mu t^{4}+3\right) t^{2}},
$$

$$
x=-6 \tau+\frac{\left(3 \tau^{2}+\mu\right)\left(3 \mu \tau^{2}+1\right)}{\left(\mu \tau^{2}+3\right) \tau},
$$

where $\tau=t^{2}$. This is a rational curve of the sixth order. So then, the cuspss of a system of $\Delta^{*}$ 's having a common inscribed ellipse lie on a rational curve of the sirth orrler.

The cusp-tangent of any cusp of the system is obtained by eliminating $x$ between equations (10) and (1). The result is

$$
2 \mu \tau^{4}-(\mu x-3 y) \tau^{3}+(\mu y-3 x) \tau-2 \mu=0,
$$

$$
2 \mu\left(\tau^{4}-1\right)-\left(\mu \tau^{2}+3\right) \tau x+\left(\mu+3 \tau^{2}\right) \tau y=0,
$$

where $\tau \equiv t^{2}$. What this line envelopes as $\alpha$ varies may be seen by the introduction of Cartesian coïrdinates, but a simpler method, though it is somewhat artificial, is as follows: Take an ellipse in the form

$$
x=\tau-\frac{\mu}{3 \tau} .
$$

The clinant of a normal to this ellipse at any point $r$ is

$$
-\frac{d x}{d y}=-\frac{\frac{d x}{d \tau}}{\frac{d y}{d \tau}}=\frac{3 \tau^{2}+\mu}{\mu \tau^{2}+3},
$$

hence the equation of this normal is

$$
2 \mu\left(\tau^{4}-1\right)-\left(\mu \tau^{2}+3\right) x \tau+\left(3 \tau^{2}+\mu\right) y \tau=0,
$$


which is obviously the same as equation (13). Hence, the cusp-tangents of a system of $\Delta^{5}$ 's having the same orthoptic curve are normals of a concentric ellipse; and, therefore, their envelope is the evolute of this ellipse.

This is the second ellipse which we have found common to the $\Delta^{5 \prime} \mathrm{s}$ of the system.

I. Curve for $\mu=1$.

§6. Some special cases.

The particular pentadeltoid for which $\mu=1$ stands out as a curve of special interest on account of the behavior of its nodes and odd tangents. Here equation (6) becomes

$$
\kappa t_{1}^{3}-\kappa^{3} t_{1}^{3}+t_{1}^{2}-\kappa^{2}=0
$$

or

$$
\left(t_{1}^{2}-\kappa^{2}\right)\left(\kappa t_{1}^{3}+1\right)=0,
$$

whose roots are obviously $\pm \kappa$ and the three cube roots of $-1 / \kappa$. But $t_{1}^{2} \equiv t$ and $\kappa^{2} \equiv-\alpha$, therefore the first two roots are simply equivalent to $t=-\alpha$ in the equation

$$
t^{5}+\alpha t^{4}+x t^{3}+\alpha y t^{2}+t+\alpha=0,
$$

whence we have as the equation of the two odd tangents

$$
x-y=0 \text {, }
$$

the axis of reals. In this case therefore two of the odd tangents coincide and remain fixed as a varies. In fact, by putting $\mu=1$ in equation (4), we find that two of the nodes are the fixed points \pm 2 , whatever the value of $\alpha$.

Putting the three other roots of $(6 a)$ into the equation of the curve on which the nodes lie, that is in

we obtain

$$
4 x=-\frac{\kappa^{3}}{t_{1}^{3}}+6 \frac{\kappa}{t_{1}}+i \frac{t_{1}}{\kappa},
$$

$$
\begin{aligned}
& 4 x_{1}=\tau^{3}-6 \tau-\frac{3}{\tau}, \\
& 4 x_{2}=\tau^{3}-6 \omega \tau-\frac{3 \omega^{2}}{\tau}, \\
& 4 x_{3}=\tau^{3}-6 \omega^{2} \tau-\frac{3 \omega}{\tau},
\end{aligned}
$$

where $\tau, \omega \tau$, and $\omega^{2} \tau$ are the three cube roots of $\kappa^{1}$. Now the odd tangents from these nodes are readily obtained, since a point and a clinant of each is known. The clinants are

$$
-\frac{\alpha}{t}=\frac{\kappa^{2}}{t_{1}^{2}}=\stackrel{B}{V} \kappa^{\ddot{y}}=\tau^{2}, \omega^{2} \tau^{2}, \omega \tau^{2} .
$$


Hence the tangents are:

$$
\begin{aligned}
& x-\tau^{2} y=\tau^{3}-\frac{1}{\tau}, \\
& x-\omega^{2} \tau^{2} y=\tau^{3}-\omega^{2}, \\
& x-\omega \tau^{2} y=\tau^{3}-\frac{\omega}{\tau},
\end{aligned}
$$

These three odd tangents evidently pass through the same point $\tau^{3}$ and are equally spaced. This may be stated thus:

When $\mu=1$, three of the odd tangents pass through a point and are equally spaced. (See Fig. 3.)

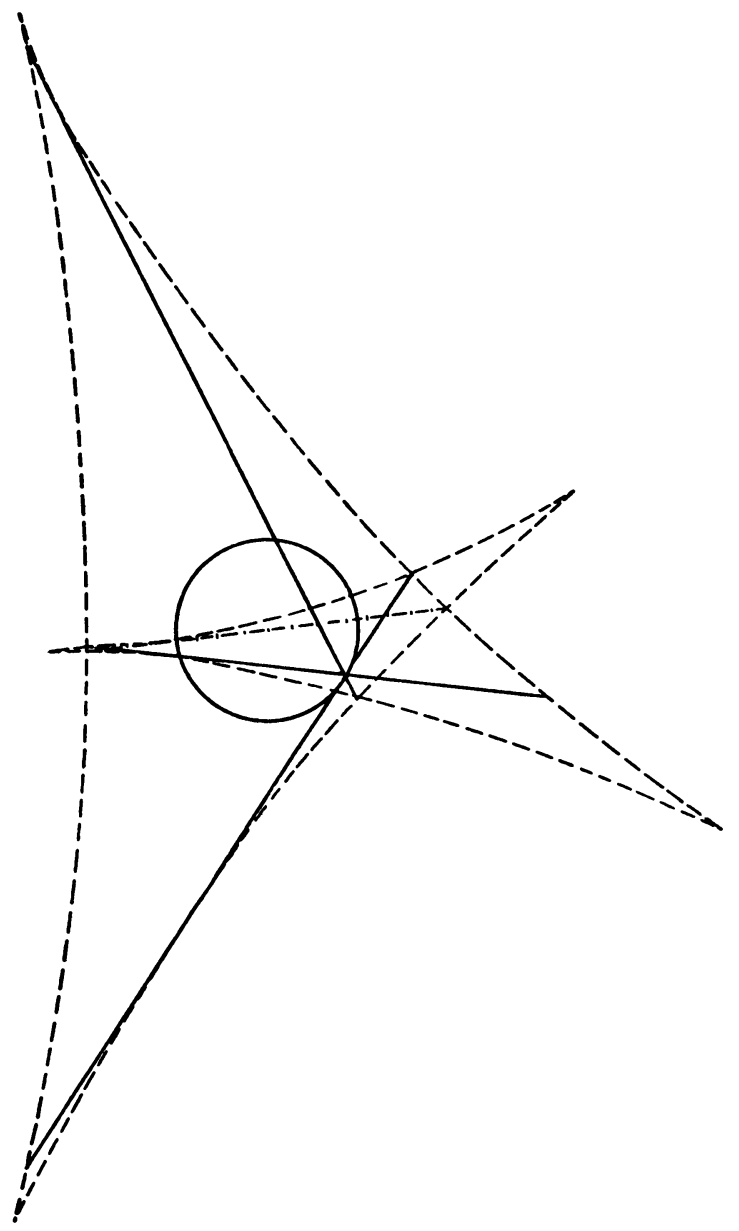

FiG. 3. Pentadeltoid when $\mu=1$. 
Since $\tau^{3}=\kappa^{4}=\alpha^{2}$, the point $\tau^{3}$ is

$$
x_{1}=\alpha^{2},
$$

which for varying $\alpha$ traces out the unit circle. The point $\alpha^{2}$ is an equiangular point of the triangle formed by the three nodes $x_{1}, x_{2}$, and $x_{3}$, and may be shown to be the positive one. It follows that the triangle formed by the three variable nodes is such that as a varies, its vertices trace out the parastroid

$$
4 x=\tau^{3}-6 \tau-\stackrel{3}{\tau},
$$

and its positive equiangular point moves on the unit circle.

From the point $\alpha^{2}$, there will be two tangents to the $\Delta^{5}$ other than the odd tangents mentioned above. The parameters of these are obtained by putting $x=\alpha^{2}$ in equation $(1 a)$. In the resulting equation

$$
\left(t^{2}+\alpha t+\alpha^{2}\right)\left(t^{3}+\begin{array}{l}
1 \\
\alpha
\end{array}\right)=0,
$$

the second factor gives the tangents already found. The roots given by the first factor are

$$
t_{1}=\omega \alpha \quad \text { and } \quad t_{2}=\omega^{2} \alpha .
$$

These values of $t$, substituted in $(1 a)$, give as the equation of the two tangents wanted :

$$
x+\omega^{2} y=\alpha^{2}+\begin{gathered}
\omega^{2} \\
\alpha^{2},
\end{gathered}, \quad x+\omega y=\alpha^{2}+\begin{aligned}
& \omega \\
& \alpha^{2}
\end{aligned},
$$

two lines whose directions are independent of the value of $\alpha$, and which, together with the axis of imaginaries, form an equilateral triangle. As a varies, these turo tangents through the point $\alpha^{2}$ but not through nodes move parallel to themselves and together with the axis of imaginaries form an equilateral. triangle.

II. Curve for $\mu=3$.

This special case is of interest on account of its cusps and its cusp-tangents. The equations giving the parameters of the cusps can be solved when $\mu=3$. For convenience, however, we shall take $\mu=-3$, that is, revolve our curve through an angle $\pi / 2$. Then equation (10) becomes

$$
3\left(t^{5}-\alpha t^{4}-t+\alpha\right)=0,
$$

or

$$
\left(t^{4}-1\right)(t-\alpha)=0,
$$

of which the roots are $\alpha, \pm 1$ and $\pm i$. The cusps are obtained by putting 
these values of $t$ in the map equation of the curve (2) when $\mu=-3$. Thus the five cusps are:

$$
\begin{gathered}
x_{1}^{\circ}=3 x^{2}-\frac{1}{\alpha^{2}}, \\
x_{2}=6-8 \alpha, \quad x_{4}=-6-8 i \alpha, \\
x_{3}=6+8 x, \quad x_{5}=-b+8 i \alpha .
\end{gathered}
$$

The cusp-tangent at the cusp $x_{1}$ is found by putting $t=-\alpha$ in the line equation of the $\Delta^{5}$, that is, in

$$
t^{5}-3 \alpha t^{4}+x t^{3}+\alpha y t^{2}-3 t+\alpha=0 .
$$

It is

$$
x+y=2\left(\alpha^{2}+\begin{array}{c}
1 \\
\alpha^{2}
\end{array}\right),
$$

a line which is always parallel to the axis of imaginaries, since its clinant is independent of $\alpha$. The cusp itself, as $\alpha$ varies, traces out an ellipse whose equation is

$$
x=3 \alpha^{2}-\frac{1}{\alpha^{2}},
$$

which is recognized as the inscribed ellipse (3) when $\mu=-3$. Hence, when $\mu=-3$, one cusp of the $\Delta^{s}$ moves on the inscribed ellipse, while the cusptangent is always perpendicular to the axis of reals.

The cusp-tangents at $x_{2}$ and $x_{3}$ are *

$$
x+\alpha y=2+2 \alpha, \quad x-\alpha y=2-2 \alpha,
$$

two lines which are perpendicular to each other, and which always pass through the point 2, whatever the value of $\alpha$. From the values of $x_{2}$ and $x_{3}$ given above it is evident that the two cusps themselves move along a circle of radius 8 with center at the point 6 , as a varies; in fact, they are opposite extremities of a diameter of this circle (see Fig. 4).

In a similar manner it is found that the other cusp-tangents are

$$
x-i \alpha y=-2+2 i \alpha, \quad x+i \alpha y=-2-2 i \alpha,
$$

two lines which pass through the point -2 , and which are perpendicular to each other, for all values of $\alpha$. These two cusps are opposite extremities of a diameter, tracing out the circle of radius 8 and center - 6, (see Fig. 4).

When $\mu=-3$, therefore, the five cusps trace out the curves:

* Obtained by substituting $t= \pm 1$ in equation ( $1 b)$. 


$$
\begin{aligned}
& x=3 \alpha^{2}-\frac{1}{\alpha^{2}} \cdots \text { an ellipse; } \\
& x=6+8 \alpha \cdots \quad \text { a circle; } \\
& x=-6+8 \alpha \cdots \text { a circle. }
\end{aligned}
$$

The ellipse (14), to which the cusp-tangents are normal, here degenerates into the segment

$$
x=\tau+\frac{1}{\tau}
$$

to which one cusp-tangent is always perpendicular, and through the extremities of which (the points \pm 2 ), the four other tangents pass in pairs.

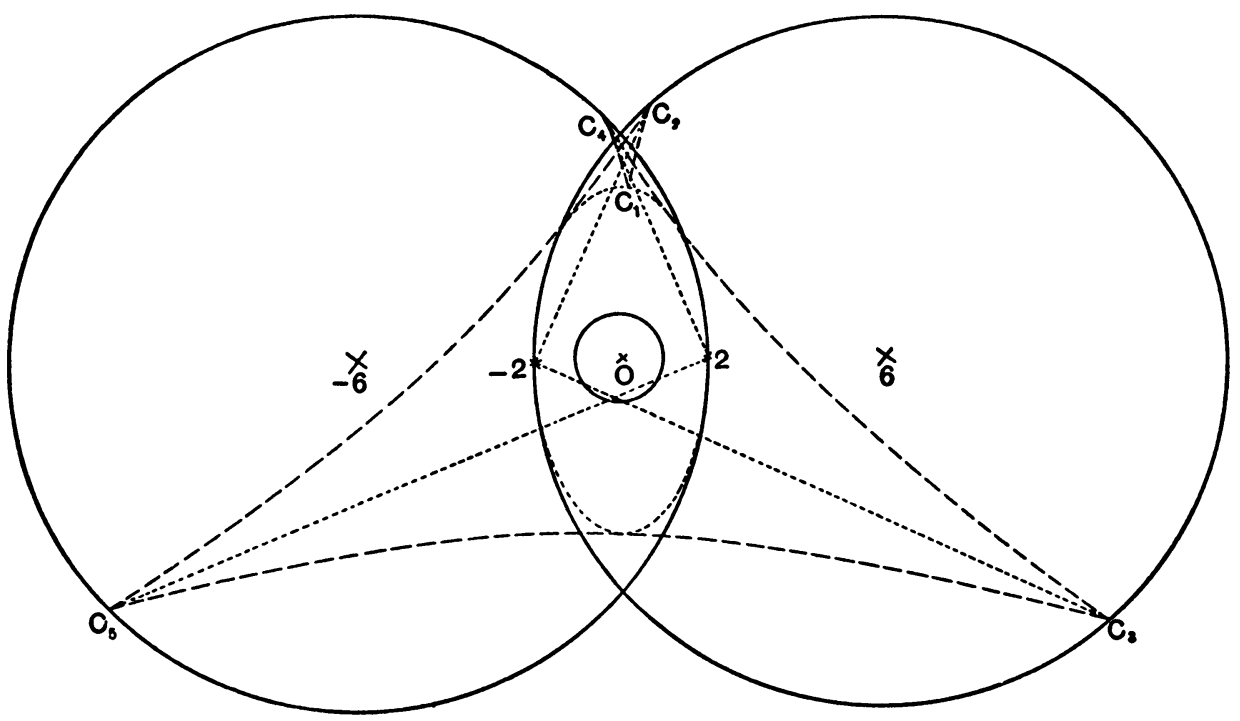

Fig. 4. Pentadeltoid when $\mu=-3$.

§ 7. Three ellipses common to the system.

Two ellipses which are common to all $\Delta^{5}$ 's of the system (1), have already been found. The first is the orthoptic ellipse

$$
x=-\left(t^{2}+\frac{\mu}{t^{2}}\right)
$$

or

$$
x=\tau+\frac{\mu}{\tau}
$$


the second is an ellipse to which all the cusp-tangents of the system are normal. The equation of this second ellipse is

$$
x=\tau-\frac{\mu}{3 \tau} \text {. }
$$

A third ellipse arises in the following manner: A line cuts the $\Delta^{5}$ in six points, whose parameters are found by substituting in the equation of a line,

$$
\xi x+\eta y-1=0
$$

values of $x$ and $y$ from the map equation of the $\Delta^{5}$,

$$
x=-\left(3 t^{2}+2 \alpha \mu t-\frac{\mu}{t^{2}}-\frac{2 \alpha}{t^{3}}\right) .
$$

Thus a sextic in $t$ is obtained, whose equation is

$$
-2 \frac{\eta}{\alpha} t^{6}+(3 \xi-\mu \eta) t^{5}+2 \alpha \mu \xi t^{4}+t^{3}+2 \mu \frac{\eta}{\alpha} t^{2}+(3 \eta-\mu \xi) t-2 \alpha \xi=0,
$$

which may be represented symbolically by the form

$$
\left(\alpha_{1} t+\alpha_{2}\right)^{6} \equiv\left(\beta_{1} t+\beta_{2}\right)^{6}=0 .
$$

The six roots of this sextic will be a self-apolar set if

$$
\left(\alpha_{1} \beta_{2}-\alpha_{2} \beta_{1}\right)^{6}=0 \text {. }
$$

Putting the coefficients of the sextic into this apolarity condition, we obtain

or

$$
4 \xi \eta-\frac{1}{6}(3 \xi-\mu \eta)(3 \eta-\mu \xi)+\frac{4}{15} \mu^{2} \eta \xi-\frac{1}{40}=0,
$$

$$
4(5 \xi+\mu \eta)(5 \eta+\mu \xi)=1,
$$

the line equation of a conic. Since $\xi$ and $\eta$ appear symmetrically, the curve is symmetrical with respect to the two axes and its center is the origin. Hence by making $\xi=\eta$, we find the points in which the conic cuts the real axis. They are $\pm(5+\mu)$. Similarly by making $\xi=-\eta$, we have the intercepts on the axis of imaginaries. They are $\pm i(5-\mu)$. These four points are actual intersections, therefore the conic is an ellipse whose map equation is easily seen (from its intersections on the axes) to be

$$
x=5 \tau+\frac{\mu}{\tau} .
$$

The geometrical significance of the parameters being self-apolar is that the tangents of the curve at the six points on a line cut the line at infinity in six 
self-apolar points. Hence, lines which cut the $\Delta^{5}$ in six points, the tangents at which cut the line at infinity in a self-apolar set, envelope a concentric ellipse which is the same for all $\Delta^{5}$ 's of the system having a common inscribed ellipse.

In general lines which cut a $\Delta^{2 n-1}$ in points whose parameters are a selfapolar set envelope a concentric ellipse. This ellipse is not the same, however, for each $\Delta^{2 n-1}$ of the system having the same orthoptic curve if $n>3$. If $\mu=0$, then $(16)$ becomes

$$
x=5 \tau,
$$

the equation of a circle through the cusps. If $\mu=5$, equation (15) becomes the equation of the two points \pm 10 , through which we have two pencils of

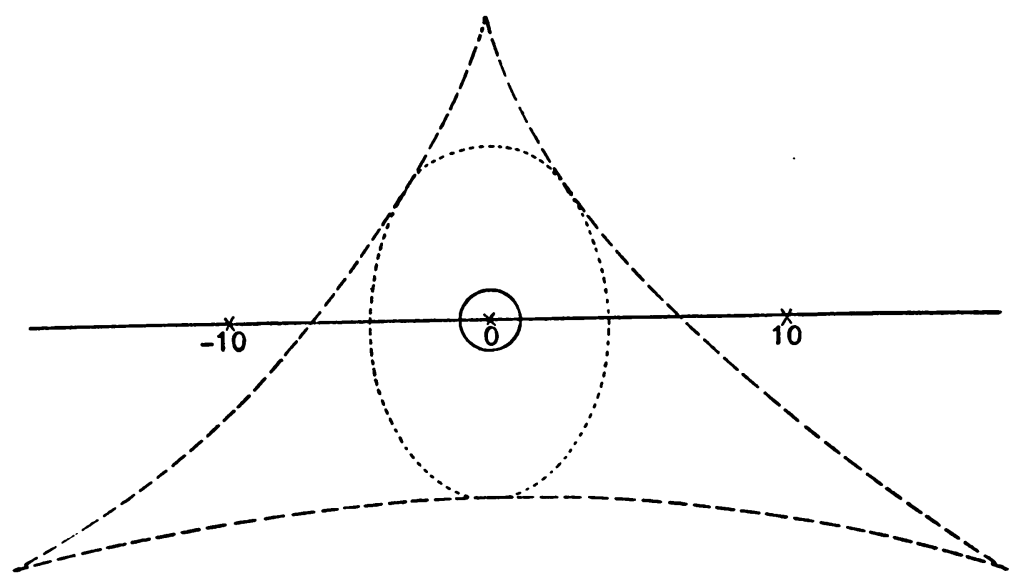

FI(;. 5. Pentadeltoid when $\mu=: 5$.

lines cutting the $\Delta^{s}$ in six points whose parameters are a self-apolar set (see Fig. 5).

This third ellipse of the $\Delta^{5}$ is the same as the second ellipse of the evolute of the $\Delta^{s}$. This can be easily shown.

A normal to the $\Delta^{i}$ is evidently

$$
-a t^{3}+\alpha y t^{2}=t^{3}\left(3 t^{2}+2 \alpha \mu t-\begin{array}{c}
\mu \\
t^{2}
\end{array}-\begin{array}{c}
2 \alpha \\
t^{3}
\end{array}\right)-\alpha t^{2}\left(\begin{array}{c}
3 \\
t^{2}
\end{array}+\frac{2 \mu}{\alpha t}-\mu t^{2}-\frac{2 t^{3}}{\alpha}\right),
$$

whence

$$
5 t^{3}+3 x \mu t^{4}+x t^{3}-x y t^{2}-3 \mu t-5 x=0,
$$

the equation of another $\Delta^{5}$, whose orthoptic ellipse is

$$
x=-\left(5 t^{2}-\begin{array}{c}
3 \mu \\
t^{2}
\end{array}\right)
$$


and whose second ellipse is

$$
x=-\left(5 t^{2}+\begin{array}{c}
\mu \\
t^{2}
\end{array}\right), \quad \text { or } \quad x=5 \tau+\stackrel{\mu}{\tau} .
$$

Comparing this with equation (16) we see it is the same as the ellipse which we have called the third ellipse of the original $\Delta^{5}$; thus the theorem is proved.

Incidentally, we have proved above that the evolute of $a \Delta^{5}$ is another $\Delta^{5}$. So, in general, the evolute of $a \Delta^{2 n-1}$ is another $\Delta^{2 n-1}$.

JOHNS HOPKINS UNIVRRSITY,

March, 1905. 